OPEN ACCESS

Edited by:

Manuel Asorey,

University of Zaragoza, Spain

Reviewed by:

Yilun Shang,

Northumbria University,

United Kingdom

Yuriy Golovaty,

Lviv University, Ukraine

Pavel Kurasov,

Stockholm University, Sweden

*Correspondence:

Luiz A. Manzoni

manzoni@cord.edu

Specialty section:

This article was submitted to

Mathematical Physics,

a section of the journal

Frontiers in Physics

Received: 29 March 2019

Accepted: 01 July 2019

Published: 25 July 2019

Citation:

Calçada M, Lunardi JT, Manzoni LA Monteiro W and Pereira M (2019) A

Distributional Approach for the

One-Dimensional Hydrogen Atom.

Front. Phys. 7:101

doi: 10.3389/fphy.2019.00101

\section{A Distributional Approach for the One-Dimensional Hydrogen Atom}

\author{
Marcos Calçada ${ }^{1}$, José T. Lunardi ${ }^{1}$, Luiz A. Manzoni ${ }^{2 *}$, Wagner Monteiro ${ }^{3}$ and \\ Marciano Pereira ${ }^{1}$
}

\begin{abstract}
${ }^{1}$ Departamento de Matemática e Estatística, Universidade Estadual de Ponta Grossa, Ponta Grossa, Brazil, ${ }^{2}$ Department of Physics, Concordia College, Moorhead, MN, United States, ${ }^{3}$ Departamento de Matemática, Universidade Federal de São Carlos, São Carlos, Brazil
\end{abstract}

We consider the one-dimensional Hydrogen atom, with the Coulomb interaction $V(x)=\frac{\gamma}{|x|}(\gamma<0)$, and use Schwartz's theory of distributions to address the non-integrable singularity at the origin. This singularity renders the interaction term $V(x) \psi(x)$ in the Schrödinger's equation, where $\psi(x)$ is the wave function, an ill-defined product in the ordinary sense. We replace this ill-defined product by a well-defined interaction distribution, $S[\psi, V](x)$, and by imposing that it should satisfy some fundamental mathematical and physical requirements, we show that this distribution is defined up to a 4-parameter family of contact interactions, in agreement with the method of self-adjoint extensions. By requiring that the interaction distribution be invariant under parity, we further restrict the 4-parameter family of interactions to the subfamily of all the parity invariant Coulomb interactions. Finally, we present a systematic study of the bound states within this subfamily, addressing the frequently debated issues of the multiplicity and parity of the bound states, and the boundedness of the ground state energy.

Keywords: one-dimensional quantum mechanics, singular interactions, contact interactions, Coulomb interaction, one-dimensional Hydrogen atom, Schwartz's distribution theory, parity invariance

\section{INTRODUCTION}

The one-dimensional (1D) hydrogen atom, with a Coulomb-like interaction ${ }^{1}$, is defined by the Hamiltonian (throughout this paper we use Rydberg atomic units $\frac{\hbar}{2 m}=1$ )

$$
H=-\frac{d^{2}}{d x^{2}}+\frac{\gamma}{|x|}, \quad x \neq 0, \quad \gamma<0,
$$

and it has been a source of considerable interest since the paper by Loudon [1], who investigated this problem due to its relevance for the physics of excitons in strong magnetic fields. The 1D Coulomb potential finds applications in several fields, such as the quasi one-dimensional hydrogen atom in astrophysical systems with very strong magnetic fields [2], in quantum wires and carbon nanotubes (see, e.g., [3-5] and references therein), etc.-for a recent review of the applications of the 1D Coulomb interaction see Loudon [6].

Despite its many applications and deceptive simplicity, the $1 \mathrm{D}$ hydrogen atom has been a source of great controversy in the literature. This is due to the fact that the 1D Coulomb potential has a non-integrable singularity at the origin, rendering its mathematical treatment non-trivial. In

\footnotetext{
${ }^{1}$ It should be noticed that the potential in Equation (1), $V(x)=\frac{\gamma}{|x|}$, is not the solution of the 1D Maxwell's equations for a
} point source, see for example [7,8]. Nevertheless, we follow the practice in the literature and call it the 1D Coulomb potential. 
particular, the singularity at the origin makes it unclear what are the boundary conditions (b.c.) to be used there and, consequently, whether the potential is impenetrable or not at the singularity (see, e.g., [7]). This singularity also obscures the properties of the system under parity transformations, since the behavior of $V(x)=\frac{\gamma}{|x|}$ on $\mathbb{R} \backslash\{0\}$ is not sufficient to ensure that the system is parity invariant (see section 3 for further details).

Several treatments, including Loudon's [1], regularize the potential by introducing a small cutoff $a$ via $V(x)=\gamma /(|x|+a)$, and taking the limit $a \rightarrow 0$ at the end. This regularization procedure has led to conflicting results in which concerns the degeneracy or not of the spectrum, the existence of even wave functions and whether the associated spectrum is continuous or discrete, and the stability of the model (unboundedness of the ground state energy from below, in the $a \rightarrow 0$ limit) $[1,6,9,10]$. This is, perhaps, unsurprising, since regularization procedures need to be employed with great care and often need additional input, such as symmetry, to yield meaningful results, as is well-known in quantum field theory [11] or in the study of chemical indices $[12,13]$ - see $[14,15]$ for rigorous treatments of the regularized 1D Coulomb potential, and [16] for a general treatment of regularized Sturm-Liouville operators.

Many other approaches, such as the use of generalized Laplace transform [17], Fourier transform [18], superpotentials [8], among others [19-23], have also been employed in the solution of the $1 \mathrm{D}$ hydrogen atom. Still, these either have not addressed or have not been able to unequivocally resolve all the issues above mentioned. Recognizing that the Hamiltonian corresponding to (1) is symmetric, but not self-adjoint, has led to the application of the rigorous theory of self-adjoint extensions (SAE) of symmetric operators to this problem by several authors [7, 24-27], who have shown that the Hamiltonian (1) is not essentially self-adjoint and, therefore, does not have a unique self-adjoint extension [28]. In fact, it has been shown in the SAE approach that this Hamiltonian admits a four-parameter family of extensions [7, 29].

Despite SAE's rigorous and unequivocal results, confusion still persists in the literature, particularly concerning the parity of the solutions and the boundedness or not of the ground state energy. In addition, although the method of SAE clarifies the possible extensions of the Hamiltonian, it cannot decide which extension (or b.c.) is the physically sensible one. Thus, a more physically appealing, albeit still rigorous, alternative method is certainly desirable and may help to shed light on some of the open problems remaining in the field. With this in mind, in section 2 we revisit the $1 \mathrm{D}$ Coulomb interaction and address the singularity using the Schwartz's theory of distributions, thus generalizing the method recently developed for $1 \mathrm{D}$ contact interactions [30] (also see [31]) to include long-range singular interactions. In this approach we deal with the non-integrable singularity of the potential at the origin by replacing the (generally) ill defined product $V(x) \psi(x)$ in the Schrödinger equation by a well-defined interaction distribution $S[\psi, V]$, to be determined from fundamental mathematical and physical requirements. Such requirements are imposed on the interaction distribution in order that fundamental postulates of quantum mechanics, such as the superposition principle and probability conservation, be satisfied also in the singular case. The interaction distribution is, essentially, a distributional regularization of the (ill-defined) term $V(x) \psi(x)$ and, by a general result of the distribution theory [32], it is determined only up to a sum of contact interactions at the singularity (that is, the Dirac delta and it's derivatives). We show that the requirements imposed on the interaction distribution reduce the contact terms to a four parameter family of point interactions, thus agreeing with the SAE results [7, 29]. It should be noticed that Kurasov used distributional methods to address singular potentials, including the 1D Coulomb potential [24]. However, Kurasov uses distribution theory in the context of the self-adjoint operator theory, in order to obtain the particular Friedrichs extension of the symmetric operator (1), also see [25, $33,34]$. Our approach is fundamentally different in which here the only requirements are from within the distribution theory and simple physical requirements from quantum theory. In addition, an explicit general form for the interaction distribution $S[\psi, V]$ is constructed, including all interactions of the fourparameter family of contact interactions, a feature that renders the distributional approach particularly suitable for symmetry analyses [30, 31].

We argue that, given the parity invariance of the original Coulomb potential on $\mathbb{R} \backslash\{0\}$, it is natural to impose the same property for the interaction distribution-we, again, stress that the parity invariance of the potential $V(x)$ on $\mathbb{R} \backslash\{0\}$ is not sufficient to ensure that the interaction, defined on the entire $\mathbb{R}$, is invariant: the behavior of the contact interactions must also be taken into account. Thus, in section 3 we show that requiring parity invariance further reduces the allowed interactions to either a two-parameter interaction (permeable origin) or an one-parameter interaction (impermeable origin) [30]. We then present, in section 4, a systematic study of the bound states for the subfamily of all parity invariant interactions, addressing questions that are at the origin of the controversies about the 1D hydrogen atom in the literature, such as the multiplicities of bound state energies, the parity of the corresponding eigenfunctions and the boundedness of the ground state (see $[6,7,26]$ and references therein)-as far as we know, such a systematic investigation, including all possible parity invariant b.c., is missing in the literature and it may help to clarify the controversies mentioned above. The results are discussed in section 5 and two appendices, on essentials of distribution theory and the solutions of the Whittaker equation, are included for convenience.

\section{A DISTRIBUTIONAL APPROACH TO THE 1D HYDROGEN ATOM INTERACTION}

The time independent 1D Schrödinger equation

$$
-\frac{d^{2}}{d x^{2}} \psi(x)+V(x) \psi(x)=E \psi(x)
$$

introduces the interaction of a quantum particle with an external potential $V(x)$ via the product $V(x) \psi(x)$, which is well-defined for potentials described by regular distributions. However, for 
singular potentials, such as the (attractive) 1D Coulomb potential

$$
V(x)=\frac{\gamma}{|x|}, \quad x \neq 0, \quad \gamma<0,
$$

the product $V(x) \psi(x)$ is ill-defined, due to the singularity of the potential at the origin. We will address this difficulty by considering the Schrödinger equation in the distributional sense, that is, every term in Equation (2) must correspond to a distribution. This requires particular attention to the product term $V(x) \psi(x)$, not only due to the singularity of the $1 \mathrm{D}$ Coulomb potential, but also because the naive product of two distributions is not necessarily a well-defined distribution.

\subsection{The 1D Coulomb Potential as a Distribution}

The 1D Coulomb potential, Equation (3), has a (Lebesgue) non-integrable singularity at the origin (its integral diverges logarithmicaly near the origin). As a consequence, it does not define a regular distribution ${ }^{2}$ on any open interval including the origin. However, it defines a regular distribution on any open interval which does not include the origin, such as $\mathbb{R} \backslash\{0\}$. Any distribution defined on the entire real line and which coincides with $V(x)$ on $\mathbb{R} \backslash\{0\}$ [that is, a distributional regularization of $V(x)]$ will necessarily be singular, and it will not be unique. In fact, the difference between any two distributions satisfying this requirement [i.e., between two distributional regularizations of $V(x)]$ is a distribution concentrated at the origin, hence given by a linear combination of the delta distribution and its derivatives up to (and including) the greatest order of the two distributions (see the Theorem in Appendix 1).

Let $r_{c}$ be the order of any distribution $V_{c}(x)$ defined on the entire real line and coinciding with $V(x)=\frac{\gamma}{|x|}$ on $\mathbb{R} \backslash\{0\}$. Then, the minimum possible value for $r_{c}$ is zero. To see this, observe that on $\mathbb{R} \backslash\{0\}$ any primitive of $V_{c}(x)$ must have the general form $V_{c}^{(-1)}(x)=\gamma \operatorname{sgn}(x) \log |x|+c_{1}+c_{2} \theta(x)$, with $c_{1}$ and $c_{2}$ arbitrary constants and $\theta$ the Heaviside theta distribution ${ }^{3}$ (see Appendix 1 for the notation). The function $V_{c}^{(-1)}(x)$ diverges logarithmically as $x \rightarrow 0^{ \pm}$, but it is integrable around the origin and, thus, it must be the derivative of a continuous (but not differentiable at $x=0$ ) function. It follows that $V_{c}^{(-1)}=$ $W^{(1)}(x)$, with

$$
W(x)=\gamma|x|(\log |x|-1)+c_{1} x+c_{2} t(x)+c_{3},
$$

where $t(x)=x \theta(x)$ is a continuous function [a primitive of $\theta(x)]$ and $c_{3}$ is another arbitrary constant. We have, for instance, that $W(0) \equiv \lim _{x \rightarrow 0} \gamma|x|(\log |x|-1)+c_{3}=c_{3}$. Therefore, we have found a continuous function $W(x)$, not differentiable at the origin, whose second distributional derivative is a distribution $V_{c}(x)$ which coincides with $\frac{\gamma}{|x|}$ on $\mathbb{R} \backslash\{0\}$. Hence, we conclude that $W(x)$ has order -2 and, consequently, $V_{c}(x)=W^{(2)}(x)$ has order zero (see Appendix 1). Any other distribution coinciding with

\footnotetext{
${ }^{2}$ For a definition of the main terms of distribution theory used in this work refer to the Appendix 1.

${ }^{3}$ Notice that it is possible to have different arbitrary constants on opposite sides of the origin.
}

$\frac{\gamma}{|x|}$ on $\mathbb{R} \backslash\{0\}$, and also having order zero, must differ from $V_{c}(x)$ only by a multiple of the delta distribution (see the Theorem in Appendix 1).

\subsection{The Interaction Distribution}

Let us now, following the approach introduced in Calçada et al. [30], substitute the ill-defined product $V(x) \psi(x)$ in the time independent Schrödinger equation by a well-defined distribution $S[\psi, V]^{4}$. Then, the distributional Schrödinger equation takes the form:

$$
\psi^{\prime \prime}+E \psi=S[\psi, V]
$$

The interaction distribution $S[\psi, V]$ must be determined from fundamental mathematical and physical requirements, namely,

R1. The distribution $S[\psi, V]$, defined on the entire real line, must coincide with $V(x) \psi(x)$ on $\mathbb{R} \backslash\{0\}$;

R2. The distribution $S[\psi, V]$ must depend linearly on the wave function $\psi$ and its derivative.

R3. The wave function must correspond to a regular distribution (i.e., to a locally integrable function in the Lebesgue sense) in any interval around the origin. Thus, its order, $r_{\psi}$, must be bounded from above by $r_{\psi} \leq-1$.

R4. The probability flux must be conserved across the origin.

The reasons why any well-defined system needs to satisfy these requirements are as follows. $\mathbf{R} \mathbf{1}$ is a purely mathematical necessity from the definition of a distributional regularization (and it is self-evident). $\mathbf{R} \mathbf{2}$ is necessary for the superposition principle to hold and in order for the dynamics to be given by Schrödinger's equation for local interactions. R3 is necessary (although not sufficient) to obtain square integrable wave functions around the origin-both $\mathbf{R} \mathbf{3}$ and $\mathbf{R 4}$ are necessary requirements of the probabilistic interpretation of quantum mechanics. Taken together, $\mathbf{R} \mathbf{1}-\mathbf{R} \mathbf{4}$ are equivalent to require self-adjointness of the Hamiltonian (see [28] for requirements similar to R3 and R4, notice that there $\mathbf{R} \mathbf{1}$ and $\mathbf{R} \mathbf{2}$ are automatically satisfied).

Equation (5), with requirement R1, applied to the 1D Coulomb interaction, implies that

$$
\begin{aligned}
\psi^{\prime \prime}+E \psi & =S[\psi, V]=\frac{\gamma}{|x|} \psi, \\
\text { for } x \in \mathbb{R} \backslash\{0\} & =(-\infty, 0) \cup(0,+\infty) .
\end{aligned}
$$

The general solution for this equation is presented in the Appendix 2, in terms of the well-known Whittaker's functions. In what follows, an important property of the solution there presented is the fact that $\psi(x)$ has finite lateral limits $\psi\left(0^{ \pm}\right)$when $x \rightarrow 0^{ \pm}$[see Equation (A6)].

Following the ideas of the previous subsection, we need to find a (ordinary) primitive of the term $\frac{\gamma}{|x|} \psi$, defined on $\mathbb{R} \backslash\{0\}$. It is not difficult to see that, by conveniently choosing the integration constants, we can obtain a primitive in the form of the following

\footnotetext{
${ }^{4}$ The notation $S[\psi, V]$ is due to the functional dependence on $V(x)$ and $\psi(x)$.
} 
ordinary function ${ }^{5}$

$$
\begin{aligned}
G[\psi, V](x)= & \gamma\left\{\psi(x) \operatorname{sgn}(x) \ln |x|-\psi^{\prime}(x)|x|(\ln |x|-1)\right. \\
& \left.-\int_{0}^{x} \psi(t)(|t| E-\gamma)(\ln |t|-1) d t\right\}
\end{aligned}
$$

where $\operatorname{sgn}(x)=\theta(x)-\theta(-x)$ stands for the sign function. It can be easily checked that the (ordinary) derivative of $G[\psi, V](x)$ coincides with the ordinary function $\frac{\gamma}{|x|} \psi$ on $\mathbb{R} \backslash\{0\}$, as desired.

It is important to notice that the function $G[\psi, V](x)$ is locally integrable and, thus, defines a distribution over the entire real line. To demonstrate this, it is enough to check the integrability in any finite interval containing the origin: the first term within the brackets in (7) is locally integrable because $\psi(x)$ is bounded (see Appendix 2) and $\ln |x|$ is integrable; the third term (the integral) defines a continuous function for all $x$, since the integrand is an integrable function, by the same reason of the first term; finally, the middle term is also integrable, since, for any fixed $R>0$ and arbitrary $\eta>0$,

$$
\begin{aligned}
\int_{\eta}^{R} \psi^{\prime}(x)|x|(\ln |x|-1) d x= & \left.|x|(\ln |x|-1) \psi(x)\right|_{\eta} ^{R} \\
& -\int_{\eta}^{R} \psi(x) \ln |x| d x
\end{aligned}
$$

and both terms in the r.h.s of this equation have well-defined limits when $\eta \rightarrow 0^{+}$. The same holds for the integral $\int_{-R}^{-\eta} \psi^{\prime}(x)|x|(\ln |x|-1) d x$.

The fact that $G[\psi, V](x)$ defines a regular distribution on the entire real axis implies that its order is $r_{G} \leq-1$. As a consequence, its distributional derivative $G^{\prime}[\psi, V](x) \equiv$ $S_{0}[\psi, V](x)$ is defined over the entire real axis, has order $\leq 0$ and, as mentioned above, coincides with the product $\frac{\gamma}{|x|} \psi(x)$ when restricted to the region $\mathbb{R} \backslash\{0\}$. Any other distribution $S[\psi, V](x)$ satisfying $\mathbf{R} 1$ must differ from $S_{0}[\psi, V](x)$ only by a distribution concentrated at the origin (a contact interaction) and, from the Theorem on Appendix 1, we have that

$$
S[\psi, V](x)=S_{0}[\psi, V](x)+\sum_{n=0}^{r} c_{n}[\psi, V] \delta^{(n)}(x),
$$

where the (complex) coefficients $c_{n}[\psi, V]$ must be linear functionals of $\psi(x)$ and its derivative, to satisfy requirement R2. From R3 and the fact that indefinite integration (taking the primitive) decreases the order by one (see Appendix 1), the order

${ }^{5}$ The ordinary function $G[\psi, V](x)$, defined on $\mathbb{R} \backslash\{0\}$, can be obtained by following a simple procedure. For $x>0$, one may start by taking the following primitive of $\frac{\gamma \psi}{|x|}$

$$
\int_{1}^{x} \frac{\gamma \psi}{|x|} d x, \quad x>0
$$

After integrating twice by parts and using the Schrödinger Equation (6) to rewrite the integrand resulting from the last integration by parts, one finds a primitive defined on $x>0$. A primitive defined on $x<0$ may be obtained in a similar way, by replacing the lower limit in the above integral by -1 and repeating the process. Finally, the function $G[\psi, V](x)$ can be obtained from these two primitives, found on each side of the origin, by choosing suitable arbitrary constants. of $S[\psi, V](x), r_{s}$, must be at most $r_{s}=+1$, since it follows from Equation (5) that the distribution $S[\psi, V](x)$ must have the same order as $\psi^{\prime \prime}(x)$ (the orders of the $\delta$ and $\delta^{\prime}$ are 0 and +1 , respectively [32]). Therefore, requirements $\mathbf{R} \mathbf{1}-\mathbf{R} \mathbf{3}$ imply that the distributional Schrödinger equation, Equation (5), with the most general interaction distribution $S[\psi, V](x)$, can be written as

$$
\begin{aligned}
\psi^{\prime \prime}(x)+E \psi(x)= & S_{0}[\psi, V](x)+c_{0}[\psi, V] \delta(x) \\
& +c_{1}[\psi, V] \delta^{\prime}(x),
\end{aligned}
$$

with $S_{0}[\psi, V](x)=G^{\prime}[\psi, V](x)$ and $G[\psi, V](x)$ given by the regular distribution defined by Equation (7). In the above equation the only undetermined quantities are the (functional) coefficients $c_{0}[\psi, V]$ and $c_{1}[\psi, V]$, which determine the contact term. Below we show that these coefficients are associated to the b.c. that the wave function and its derivative must satisfy around the origin and, thus, requirement $\mathbf{R} \mathbf{4}$ will restrict the possible choices for them. To this purpose, by taking a primitive of (9), we obtain

$$
\begin{aligned}
& {\left[\psi^{\prime}(x)-G[\psi, V](x)\right]-c_{0}[\psi, V] \theta(x)-c_{1}[\psi, V] \delta(x)} \\
& =-E \psi^{(-1)}(x)+c_{2},
\end{aligned}
$$

with $c_{2}$ an arbitrary constant. The distribution on the r.h.s of (10) is a continuous function, since its order is -2 .

The lateral and point limits of a singular distribution can be defined (if they exist) even at the singular point $[35,36]$. In particular, the lateral limits of the $\delta$-distribution are zero, as follows directly from the fact that the $\delta$ vanishes in the open intervals $(-\infty,-\epsilon)$ and $(+\epsilon,+\infty)$ for any $\epsilon>0$ (see [37], p. 64). Hence, it follows from (10) that the lateral limits of $\left[\psi^{\prime}(x)-G[\psi, V](x)\right]$ exist and are given by

$$
\begin{aligned}
& {\left[\psi^{\prime}-G\right]_{+}=c_{0}[\psi, V]-E \psi^{(-1)}(0)+c_{2}} \\
& {\left[\psi^{\prime}-G\right]_{-}=-E \psi^{(-1)}(0)+c_{2}}
\end{aligned}
$$

where we used the shorthand notation $\left[\psi^{\prime}-G\right]_{0^{ \pm}} \equiv \lim _{x \rightarrow 0^{ \pm}}\left\{\psi^{\prime}(x)-G[\psi, V](x)\right\} . \quad$ Subtracting these equations we obtain

$$
\left[\psi^{\prime}-G\right]_{0^{+}}-\left[\psi^{\prime}-G\right]_{0^{-}}=c_{0}[\psi, V],
$$

On the other hand, from Equation (7) we obtain that

$$
\left[\psi^{\prime}-G\right]_{0^{ \pm}}=\tilde{\phi}\left(0^{ \pm}\right) \equiv \lim _{x \rightarrow 0^{ \pm}} \tilde{\phi}(x),
$$

where $\tilde{\phi}(x)$ is defined as (see also [7])

$$
\tilde{\phi}(x) \equiv \psi^{\prime}(x)-\operatorname{sgn}(x) \gamma \psi(x) \ln |x| .
$$

Thus, the boundary condition (11) can be rewritten as (see $[15,25]$ for alternative proofs)

$$
\tilde{\phi}\left(0^{+}\right)-\tilde{\phi}\left(0^{-}\right)=c_{0}[\psi, V] .
$$

Now, by taking a primitive of Equation (10) we obtain, after some rearrangement,

$$
\begin{aligned}
\psi(x)-c_{1}[\psi, V] \theta(x)= & G^{(-1)}[\psi, V](x)+c_{0}[\psi, V] T(x) \\
& -E \psi^{(-2)}(x)+c_{2} x+c_{3},
\end{aligned}
$$


where $T(x)=\theta^{(-1)}(x)=x \theta(x)$ and $c_{3}$ is another arbitrary constant. The r.h.s. of the above equation is a continuous function $\left(G^{(-1)}[\psi, V](x)\right.$ is a continuous distribution, since it is a primitive of a regular distribution) and, thus, both sides of the equation have well-defined lateral limits when $x \rightarrow 0^{ \pm}$. Taking the lateral limits and subtracting the corresponding equations we obtain the second boundary condition

$$
\psi\left(0^{+}\right)-\psi\left(0^{-}\right)=c_{1}[\psi, V] .
$$

Equations $(13,14)$ give the b.c. satisfied by the wave function and its derivative around the origin in terms of the yet undefined coefficients $c_{0}[\psi, V]$ and $c_{1}[\psi, V]$ [which are, in fact, complex-valued linear functionals of $\psi(x)$ and $\left.\psi^{\prime}(x)\right]$. Hence, once specified, these coefficients will completely determine the interaction.

Let us now use the requirement $\mathbf{R} 4$, taking into account the b.c. (13) and (14), to restrict the possibilities for $c_{1}[\psi, V]$ and $c_{2}[\psi, V]$. First, notice that on $\mathbb{R} \backslash\{0\}$ the probability current can be conveniently rewritten in terms of $\tilde{\phi}(x)$ as

$$
\begin{aligned}
j(x) & =-i\left[\psi^{*}(x) \psi^{\prime}(x)-\psi(x) \psi^{\prime *}(x)\right] \\
& =-i\left[\psi^{*}(x) \tilde{\phi}(x)-\psi(x) \tilde{\phi}^{*}(x)\right],
\end{aligned}
$$

which has well-defined lateral limits around the origin. Probability current conservation now reads $j\left(0^{+}\right)=j\left(0^{-}\right)$ and it may establish (when the current does not vanish) a connection between the values of $\psi(x)$ and $\tilde{\phi}(x)$ on both sides of the origin. This, together with the b.c. (13) and (14), allows us to determine the most general form for $c_{0}[\psi, V]$ and $c_{1}[\psi, V]$. The procedure to find these coefficients is identical to that followed in reference [30], and all of the Equations (928) from Calçada et al. [30] apply to the current system by replacing $\psi^{\prime}\left(0^{ \pm}\right) \rightarrow \tilde{\phi}\left(0^{ \pm}\right)$. Thus, below we just summarize the results.

Permeable Interactions. In this case the wave function and its derivative [via $\tilde{\phi}(x)$ ] on both sides of the origin are connected (hence, the origin is permeable). These non-separated interactions $[30,38]$ are characterized by four parameters, and the interaction distribution $S[\psi, V](x)$, in Equation (9), is given by:

$$
\begin{aligned}
S[\psi, V](x) & =S_{0}[\psi, V](x)+c_{0}[\psi, V] \delta(x)+c_{1}[\psi, V] \delta^{\prime}(x),(16) \\
c_{0}[\psi, V] & =\left[c \mathrm{e}^{i \varphi} \psi\left(0^{-}\right)+\left(d \mathrm{e}^{i \varphi}-1\right) \tilde{\phi}\left(0^{-}\right)\right], \\
c_{1}[\psi, V] & =\left[\left(a \mathrm{e}^{i \varphi}-1\right) \psi\left(0^{-}\right)+b \mathrm{e}^{i \varphi} \tilde{\phi}\left(0^{-}\right)\right],
\end{aligned}
$$

where $a, b, c, d \in \mathbb{R}, a d-b c=1$ and $\varphi \in[0, \pi)$. The b.c. can be written in the matrix form as:

$$
\Phi\left(0^{+}\right)=\Lambda \Phi\left(0^{-}\right), \quad \Phi\left(0^{ \pm}\right)=\left[\begin{array}{c}
\psi\left(0^{ \pm}\right) \\
\tilde{\phi}\left(0^{ \pm}\right)
\end{array}\right], \quad \Lambda=\mathrm{e}^{i \varphi}\left[\begin{array}{ll}
a & b \\
c & d
\end{array}\right]
$$

Alternatively, the expression (16) can be rewritten in terms of $\psi\left(0^{+}\right), \tilde{\phi}\left(0^{+}\right)$by inverting the relations (19), resulting in Calçada et al. [30]:

$$
\begin{aligned}
& c_{0}[\psi, V]=\left[c \mathrm{e}^{-i \varphi} \psi\left(0^{+}\right)-\left(a \mathrm{e}^{-i \varphi}-1\right) \tilde{\phi}\left(0^{+}\right)\right], \\
& c_{1}[\psi, V]=-\left[\left(d \mathrm{e}^{-i \varphi}-1\right) \psi\left(0^{+}\right)-b \mathrm{e}^{-i \varphi} \tilde{\phi}\left(0^{+}\right)\right] .
\end{aligned}
$$

Impermeable Interactions. In this case we have a separated two-parameter family of interactions $[30,38]$, characterized by $j\left(0^{-}\right)=j\left(0^{+}\right)=0$, such that $\psi(x)$ and $\tilde{\phi}(x)$ on both sides of the origin are not connected (hence, an impermeable origin). The associated b.c. are given by

$$
\tilde{\phi}\left(0^{ \pm}\right)=h^{ \pm} \psi\left(0^{ \pm}\right)
$$

where $h^{ \pm} \in \mathbb{R} \cup\{+\infty\}$, with he interaction distribution assuming the form ${ }^{6}$

$$
\begin{aligned}
S[\psi, V](x) & =S_{0}[\psi, V](x)+c_{0}[\psi, V] \delta(x)+c_{1}[\psi, V] \delta^{\prime}(x), \\
c_{0}[\psi, V] & =\left[h^{+} \psi\left(0^{+}\right)-h^{-} \psi\left(0^{-}\right)\right]=\left[\tilde{\phi}\left(0^{+}\right)-\tilde{\phi}\left(0^{-}\right)\right],(22) \\
c_{1}[\psi, V] & =\left[\psi\left(0^{+}\right)-\psi\left(0^{-}\right)\right]=\left[\frac{1}{h^{+}} \tilde{\phi}\left(0^{+}\right)-\frac{1}{h^{-}} \tilde{\phi}\left(0^{-}\right)\right] .
\end{aligned}
$$

\section{SYMMETRY: PARITY INVARIANCE}

As we have seen in the previous section, the fundamental requirements R1-R4, which must be satisfied by all interactions, restrict the possible choices for the coefficients $c_{0}[\psi, V]$ and $c_{1}[\psi, V]$ to either a family of interactions with four independent parameters (in the permeable case) or to a family of interactions with two independent parameters (in the impermeable case). To further restrict the possible interactions we need to impose additional, physical, requirements.

A natural way to select subfamilies of interactions is by requiring that the underlying symmetries of the potential be maintained in the distributional theory. Hence, given that the Coulomb potential (3) is invariant under parity [i.e., an even function], we will require that the distribution interaction $S[\psi, V](x)$ introduced in (9) also be invariant under parity transformation. It should be noticed that, despite the fact that Coulomb's potential is an even function under parity, $S[\psi, V](x)$ does not necessarily have a definite parity. It is only for particular values of the parameters that the interaction distribution is invariant, as seen below.

${ }^{6}$ It is important to notice that choosing $h^{+}=+\infty\left(h^{-}=+\infty\right)$ implies $\psi\left(0^{+}\right)=$ $0\left[\psi\left(0^{-}\right)=0\right]$. The choice $h^{ \pm} \in \mathbb{R} \cup\{-\infty\}$ (instead of $h^{ \pm} \in \mathbb{R} \cup\{+\infty\}$ ) in these expressions does not change the b.c., resulting in the same interaction. Thus, it is enough to consider the parametrization above for $h^{ \pm}$, a choice of sign that will prove to be convenient in section 4 . 
For a regular potential $V(x)$, under a parity transformation, $\mathcal{P}: x \rightarrow-x, \psi(x) \rightarrow \psi(-x) \equiv \chi(x)$ and $V(x) \rightarrow \tilde{V}(x) \equiv$ $V(-x)$, the Schrödinger equation changes as Schiff [39]

$$
-\psi^{\prime \prime}(x)+V(x) \psi(x)=E \psi(x) \stackrel{\mathcal{P}}{\longrightarrow}-\chi^{\prime \prime}(x)+\tilde{V}(x) \chi(x)=E \chi(x) .
$$

Invariance under parity is automatically fulfilled if $V(x)$ is an even function and, thus, the transformed equation is identical to the original one. Similarly, for a regular potential, the corresponding transformation properties of the interaction distribution follow directly from the fact that, in this case, $S[\psi, V](x)=V(x) \psi(x)$, which transforms as $S[\psi, V](x) \stackrel{\mathcal{P}}{\longrightarrow} S[\psi, V](-x) \equiv \tilde{S}[\chi, \tilde{V}](x)$. Then, the interaction distribution is invariant (i.e., even) under parity if $\tilde{S}[\chi, \tilde{V}](x)=S[\chi, V](x)$, and, equivalently, the interaction distribution is odd under parity if $\tilde{S}[\chi, \tilde{V}](x)=$ $-S[\chi, V](x)$.

For a singular interaction, as it is the case of the Coulomb potential considered here, as we have seen, the product $V(x) \psi(x)$ is in general ill-defined and we must generalize the singular interaction distribution using the method employed in the last section. Then, the characterization of the properties of $S[\psi, V](x)$ under a symmetry transformation is made by extending the behavior of this distribution in the regular case to the singular one [30]. Therefore, under a transformation of parity the distributional Schrödinger equation transforms as

$$
\psi^{\prime \prime}(x)+E \psi(x)=S[\psi, V](x) \stackrel{\mathcal{P}}{\longrightarrow} \quad \chi^{\prime \prime}(x)+E \chi(x)=\tilde{S}[\chi, \tilde{V}](x),
$$

and the interaction distribution is characterized as

$$
\begin{aligned}
& \text { - Even: if } S[\psi, V](x) \stackrel{\mathcal{P}}{\longrightarrow} \tilde{S}[\chi, \tilde{V}](x)=S[\chi, V](x), \\
& \text { - Odd: if } S[\psi, V](x) \stackrel{\mathcal{P}}{\longrightarrow} \tilde{S}[\chi, \tilde{V}](x)=-S[\chi, V](x),
\end{aligned}
$$

regardless of whether the interaction is singular or regular. As expected, invariance under parity transformations requires an even $S[\psi, V](x)$.

Now we can consider the properties of the interaction distribution (9), with $c_{0}[\psi, V]$ and $c_{1}[\psi, V]$ given by (16) or (23), under a parity transformation. This can be further simplified by considering the behavior of $S_{0}[\psi, V](x)$ and the contact interactions in separate. To see how $S_{0}[\psi, V](x)$ behaves under parity, notice that, since $G[\psi, V](x)$ is a regular distribution, it can be represented by the ordinary function on the r.h.s. of (7). Thus, in the sense of ordinary functions, it follows that

$$
\begin{aligned}
G[\psi, V](-x)= & \gamma\left\{-\chi(x) \operatorname{sgn}(x) \ln |x|+\chi^{\prime}(x)|x|(\ln |x|-1)\right. \\
& \left.+\int_{0}^{x} \chi(t)(|t| E-\gamma)(\ln |t|-1) d t\right\} \\
= & -G[\chi, V](x),
\end{aligned}
$$

that is, the regular distribution $G[\psi, V](x)$ is odd under parity. As a consequence, since $S_{0}[\psi, V](x)=G^{\prime}[\psi, V](x)$ (derivative in the distributional sense), we conclude that the distribution $S_{0}[\psi, V](x)$ is even under parity: $S_{0}[\psi, V](x) \stackrel{\mathcal{P}}{\longrightarrow}$
$S_{0}[\chi, V]^{7}$. Therefore, the whole interaction term $S[\psi, V](x)=$ $S_{0}[\psi, V](x)+c_{0}[\psi, V] \delta(x)+c_{1}[\psi, V] \delta^{\prime}(x)$ will be even if, and only if, the distribution corresponding to the contact terms, $c_{0}[\psi, V] \delta(x)+c_{1}[\psi, V] \delta^{\prime}(x)$ is also even, i.e., if, and only if ${ }^{8}$

$$
c_{0}[\psi, V] \delta(-x)+c_{1}[\psi, V] \delta^{\prime}(-x)=c_{0}[\chi, V] \delta(x)+c_{1}[\chi, V] \delta^{\prime}(x) .
$$

In reference [30] it was shown that this condition is satisfied for permeable interactions if, and only if, the parameters in (16) satisfy

$$
a=d \quad \text { and } \varphi=0,
$$

thus reducing the allowed permeable interactions to a subfamily with only two independent parameters. For impermeable interactions, condition (29) is satisfied if, and only if, the parameters in (23) are given by

$$
h^{+}=-h^{-}=h, \quad \text { with } h \text { finite, or } h^{+}=h^{-}=+\infty \text {, }
$$

thus reducing the allowed impermeable interactions from two parameters to just one. Below we present a systematic study of the properties of the bound states for the subfamily of parity invariant (even) Coulomb interactions.

\section{BOUND STATES FOR PARITY INVARIANT COULOMB INTERACTIONS}

For bound states $(E<0)$, the general solution for the onedimensional Schrödinger equation with a Coulomb potential outside the origin [i.e., Equation (6)] is given by (A5), in the Appendix 2, and reproduced below

$$
\psi(x)=\mathcal{W}_{\frac{-\gamma}{\sqrt{-4 E}}, \frac{1}{2}}(\sqrt{-4 E}|x|)\left[B_{-} \theta(-x)+B_{+} \theta(x)\right] .
$$

The relationship between the constants $B_{+}$and $B_{-}$in this expression is established by the boundary conditions at the origin, which, in turn, are determined by the parameters of the interaction, according to the results of the sections 2.2 and 3 . Let us now investigate the properties of the bound states of parity invariant Coulomb interactions in both the permeable and impermeable cases.

\subsection{Permeable Interactions}

For the subfamily of even interactions with a permeable origin, the boundary conditions are given by (19), with $a=d$ and $\varphi=0$. By using the lateral limits given in the Appendix 2 by the expressions (A6) and (A8), these boundary conditions can be written as the following system of equations for the unknown coefficients $B_{+}$and $B_{-}$:

$$
\left\{\begin{array}{l}
W_{0}(E) B_{+}+\left[b F_{0}(E)-a W_{0}(E)\right] B_{-}=0 ; \\
F_{0}(E) B_{+}+\left[a F_{0}(E)-c W_{0}(E)\right] B_{-}=0,
\end{array}\right.
$$

\footnotetext{
${ }^{7}$ It is a simple task to show that, if $f(x)$ is an odd distribution, then $\left\langle f^{\prime}(x), \varphi(x)\right\rangle=$ $\left\langle f^{\prime}(-x), \varphi(x)\right\rangle[32]$ and, therefore, $f^{\prime}(-x)=f^{\prime}(x)$, i.e., the distributional derivative $f^{\prime}(x)$ is an even distribution. Similarly, if $f(x)$ is an even distribution, its distributional derivative is odd. Hence, as with ordinary functions, taking the distributional derivative changes the parity of the (resulting) distribution.

${ }^{8}$ Note that $\psi\left(0^{ \pm}\right)=\chi\left(0^{\mp}\right), \psi^{\prime}\left(0^{ \pm}\right)=-\chi^{\prime}\left(0^{\mp}\right), \delta(-x)=\delta(x)$ and $\delta^{\prime}(-x)=-\delta^{\prime}(x)$.
} 
where the functions $W_{0}(E)$ and $F_{0}(E)$ are defined in the Appendix 2. These equations allow for non-trivial solutions for $B_{ \pm}$if, and only if,

$$
b F_{0}(E)^{2}+c W_{0}(E)^{2}-2 a W_{0}(E) F_{0}(E)=0 .
$$

The values of $E$ which solve this equation determine the allowed bound states energies. Below we consider separately the cases with $b \neq 0$ or $b=0$.

\subsection{1. $b \neq 0$}

When $b \neq 0$, the allowed energies must be such that $W_{0}(E) \neq 0$. Otherwise, Equation (34) has no solution, since $F_{0}(E)$ and $W_{0}(E)$ cannot vanish simultaneously [see Equations (A10) and (A11) in the Appendix 2]. Therefore, Equation (34) can be rewritten in the form of a quadratic equation for $Q(E)=\frac{F_{0}(E)}{W_{0}(E)}$,

$$
b Q(E)^{2}-2 a Q(E)+c=0,
$$

whose solutions (obtained using the fact that $a^{2}-b c=1$ )

$$
Q^{( \pm)}(E)=\frac{a \pm 1}{b}
$$

are transcendental equations that determine the allowed binding energies - the explicit form and some of the properties of $Q(E)$ are given in the Appendix 2.

The relationship between the coefficients $B_{ \pm}$is obtained by substituting (35) into (33), obtaining

$$
B_{+}^{( \pm)}=\mp B_{-}^{( \pm)}
$$

Therefore, for each value of $E$ that solves the condition $Q^{(+)}(E)$ in (36) there is an associated eigenfunction given by (32) with $B_{+}^{(+)}=-B_{-}^{(+)}$, which is an odd function under parity. Similarly, for each energy $E$ satisfying $Q^{(-)}(E)$ in (36), there is an eigenfunction (32) with $B_{+}^{(-)}=B_{-}^{(-)}$, which is an even function under parity. Thus, in this case, the bound states have eigenfunctions with defined parity, and their binding energies are not degenerate.

It is instructive to consider a plot of the function $Q(E)$ to illustrate the structure of the binding energies and the parity of the associated bound states (even though to determine the actual binding energies one needs consider a specific choice of the parameters). In Figure 1 the two horizontal lines illustrate the two values in the r.h.s of (36), and the intersections between these lines and the graph of $Q(E)$ determine the allowed binding energies. Since $Q(E)$ is monotonic in the intervals between two successive discontinuities, as $|E|$ increases from zero the intersections will alternate between the lines-that is, the binding energies alternate between the solutions of $Q^{(+)}(E)$ and $Q^{(-)}(E)$ in (36). If $b>0(b<0)$, the intersections with the upper line correspond to the solutions of $Q^{(+)}(E)\left[Q^{(-)}(E)\right]$ in (36) and, therefore, to odd (even) eigenstates, whereas the energies associated to the intersections with the lower line are given by the solutions of $Q^{(-)}(E)\left[Q^{(+)}(E)\right]$ and, thus, correspond to even (odd) eigenstates. Therefore, as $|E|$ increases from zero we encounter a discrete set of bound states, each one having definite parity, with the parity alternating between successive states.

Finally, for any physically acceptable (finite) choice of the interaction parameters $a, b, c\left(b \neq 0, a^{2}-b c=1\right)$, the r.h.s of (36) is finite. Hence, there will always be a finite largest value of $|E|(E<0)$ for which the graph of $Q(E)$ intercepts the lower horizontal line, since $Q(E) \rightarrow-\infty$ as $E \rightarrow-\infty$ [see (A13) in the Appendix 2], corresponding to a ground state with finite binding energy and whose (definite) parity depends on the sign of $b$, as mentioned above.

\subsection{2. $b=0$}

Taking $b=0$ in (19) implies that $c$ is arbitrary and $a= \pm 1$ (with each choice of sign corresponding to a different interaction). In this case, Equation (34) is equivalent to

$$
W_{0}(E)=0 \quad \text { or } \quad c W_{0}(E)-2 a F_{0}(E)=0
$$

The first condition in $(38), W_{0}(E)=0$, gives [see Equation (A10) in the Appendix 2]

$$
E=\epsilon_{n}=-\frac{\gamma^{2}}{4 n^{2}}, \quad n=1,2,3, \cdots
$$

Substituting $W_{0}(E)=0$ into the second equation in (33), and taking into account the fact that $F_{0}\left(\epsilon_{n}\right) \neq 0$ [see Equation (A11)], we conclude that

$$
B_{+}=-a B_{-}
$$

and the corresponding eigenfunctions are odd (even) under parity transformations if $a=+1(a=-1)$.

The second solution in (38), since $W_{0}(E)$ and $F_{0}(E)$ cannot be simultaneously zero, can be rewritten in terms of $Q(E)$ as

$$
Q(E)=\frac{c}{2 a} .
$$

Substituting this condition into the first equation in (33) we obtain

$$
B_{+}=a B_{-},
$$

and the corresponding eigenfunctions are even (odd) if $a=$ $+1(a=-1)$, thus with parity opposite to the eigenfunctions associated with the condition $W_{0}(E)=0$

The values of energy that solve (40) correspond to the intersections of the curve $Q(E)$ with the horizontal line crossing the vertical axis at $\frac{c}{2 a}$ (similarly to the case $b \neq 0$ that was illustrated in Figure 1). In the intervals between any two successive discontinuities of $Q(E)$, which occur at the energies $\epsilon_{n}$, there is always exactly one such intersection, since $Q(E)$ is monotonic in these intervals. Therefore, as $|E|$ increases from zero, the allowed energies alternate between those in the set $\left\{\epsilon_{n}\right\}$ and the solutions of (40), with the parity of corresponding eigenstates alternating between even and odd.

In what concerns the ground state energy and the parity of the associated eigenstate, notice that while the set of energies $\left\{\epsilon_{n}\right\}$ is bounded from below (with the minimum energy corresponding to $n=1$ ), the set of energies given by (40) is also bounded from 


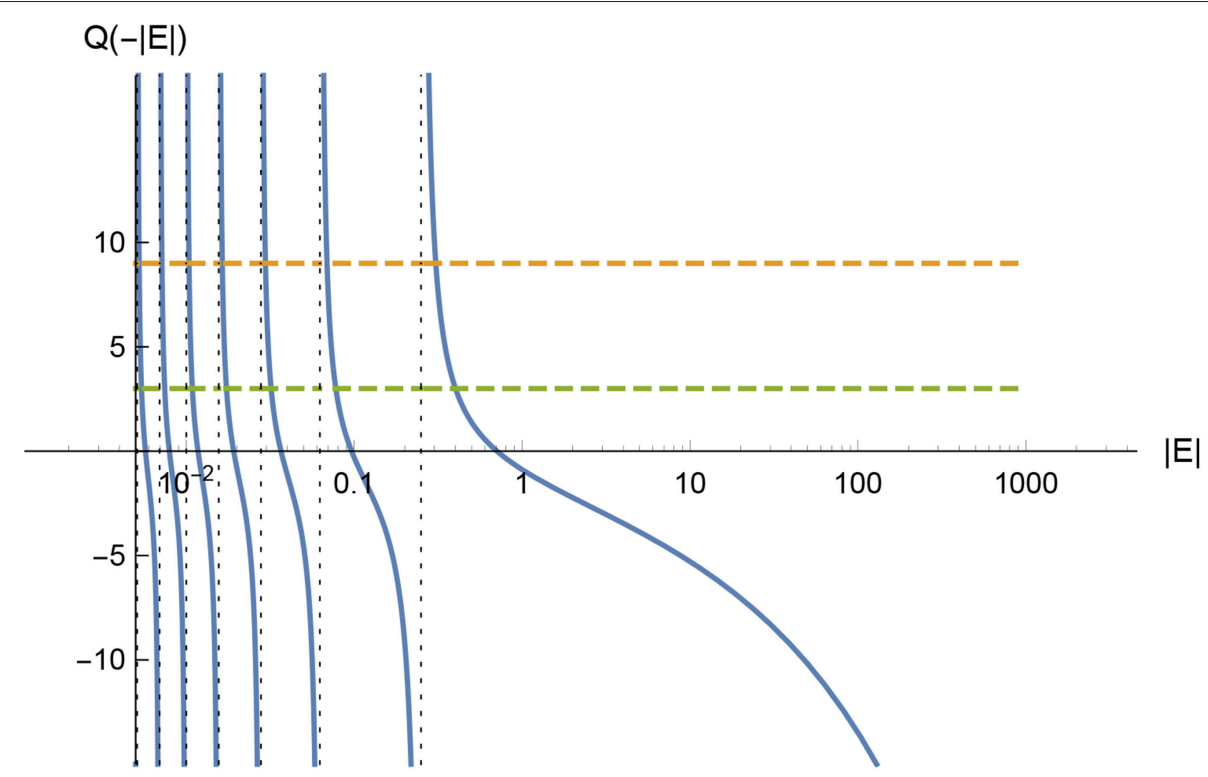

FIGURE 1 | Plot of $Q(E)$, for $E<0$, and $\gamma=-1$ (in Rydberg atomic units). The vertical dotted lines correspond to the energies $\epsilon n=-\frac{\gamma^{2}}{4 n^{2}}, n=1,2,3, \cdots$, for which $Q(E)$ is discontinuous [such that $W_{0}\left(\epsilon_{n}\right)=0$ ]. The two horizontal lines illustrate the two values on the r.h.s of the Equation $(36)$. If $b>0$ ( $\left.b<0\right)$ the upper (lower) horizontal line correspond to $Q^{(+)}(E)$ [and $\left[Q^{(-)}(E)\right.$ in Equation (36) and to the respective b.c. in Equation (37). Since $Q(E)$ is monotonic between two discontinuity points, as the $|E|$ increases the intersections of $Q(E)$ with the horizontal lines alternate between the two lines.

below, since $c / 2 a$ is finite and $Q(E) \rightarrow-\infty$ when $E \rightarrow-\infty$ (see Appendix 2). Therefore, the ground state energy is finite and given by the most negative energy satisfying the condition (40), and its parity is even (odd) if $a=+1(a=-1)^{9}$.

Having considered all the possibilities of bound states for a parity invariant Coulomb interaction with permeable origin, it is instructive to consider a few examples that illustrate these results, before proceeding to the analysis of impermeable interactions.

\subsubsection{Examples of Permeable Interactions}

Example 1. First, let us consider the case $a=+1, b=0$ and $c$ arbitrary, which corresponds to a "pure delta" interaction for the contact term in (16). In this case, the conditions for the allowed binding energies and the parity of the corresponding eigenstates are as follows.

- $W_{0}(E)=0$, with binding energies given by (39) and eigenstates that are odd under a parity transformation.

- $Q(E)=\frac{c}{2}$, with the eigenstates having even parity and the binding energies given by the set of values of $E$ such that $Q(E)$ intersects the line $c / 2$ - the ground state corresponds to the most negative (finite) energy in this set.

Example 2. Consider the so-called "delta prime" interaction for the contact term in (16), i.e., $a=+1, b \neq 0$ and $c=0$. In this case the allowed binding energies are given by the solutions of

\footnotetext{
${ }^{9}$ That the ground state energy $E_{g}$ will always belong to the set of energies satisfying (40) can be seen from the fact that, as $|E|$ increases, the last intersection between $Q(E)$ and the horizontal line $\frac{c}{2 a}$ will always occur at an energy $E_{g}<\epsilon_{1}$.
}

- $Q^{(+)}(E)=\frac{2}{b}$, corresponding to eigenstates that are odd under parity. If $b<0$, the ground state will correspond to the lowest (finite) energy of this set.

- $Q^{(-)}(E)=0$, resulting in even eigenstates. If $b>0$, the eigenstate is given by the lowest (finite) energy in this set.

Example 3. As our third example, we consider the "pure Coulomb" interaction, identified by the absence of a contact term in (16), i.e., $S[\psi, V]=S_{0}[\psi, V]^{10}$ [7] - obtained by choosing the parameters $a=1, b=c=0$. In this case the binding energies satisfy:

- $W_{0}(E)=0$, which results in odd eigenstates with eigenvalues given by (39).

- $Q(E)=0$, with the allowed energies corresponding to the intersection points of $Q(E)$ with the horizontal axis (see Figure 1), and eigenstates that are even under parity. The ground state is the lowest (finite) energy in this set.

\subsection{Impermeable Interactions}

At the end of the section 3 we saw that an impermeable interaction is even under parity if the parameters $h^{ \pm}$in (22) are such that $h^{+}=-h^{-}=h$ (for $h$ real and finite), or $h^{+}=h^{-}=$ $+\infty$. Below we consider these two cases separately.

\subsection{1. $h$ Real and Finite}

For impermeable interactions satisfying $h^{+}=-h^{-}=h$ with $h$ finite, using (A6) and (A8), the boundary conditions (22) imply

$$
\left[F_{0}(E)-h W_{0}(E)\right] B_{ \pm}=0
$$

${ }^{10}$ This interaction is sometimes identified by the so-called "periodic" boundary conditions $\psi\left(0^{+}\right)=\psi\left(0^{-}\right)$and $\tilde{\phi}\left(0^{+}\right)=\tilde{\phi}\left(0^{-}\right)$ 
which has non-trivial solutions for $B_{ \pm}$if, and only if,

$$
F_{0}(E)=h W_{0}(E) .
$$

This condition is never satisfied if $W_{0}(E)=0$, since $F_{0}(E)=$ 0 and $W_{0}(E)$ do not vanish simultaneously (see Appendix 2). Therefore, (42) can be satisfied only if $W_{0} \neq 0$, in which case it can be rewritten as a condition for $Q(E)$,

$$
Q(E)=h .
$$

For each energy satisfying this condition, the system (41) allows arbitrary solutions for $B_{+}$and $B_{-}$and, as expected, these coefficients are not connected through the origin due to the impermeability of the interaction. Thus, in this case, each binding energy is doubly degenerated. In addition, since the interaction is even, it is always possible to choose the pair of eigenfunctions associated with each energy eigenvalue to have definite parity (that is, one eigenfunction even, the other odd).

Once again, the set of allowed binding energies given by Equation (43) can be graphically visualized as the energies at which the graph of $Q(E)$ intersects the single horizontal line crossing the vertical axis at the finite height $h$ (which can be zero, positive or negative), similarly to the situation depicted in Figure 1. From the finiteness of $h$ and the fact that $Q(E) \rightarrow-\infty$ when $E \rightarrow-\infty$, it follows that the doubly degenerated ground state has finite energy - the ground state binding energy can be made arbitrarily large, by taking $h$ sufficiently large and negative, but it is still finite, since in this case $h$ is not allowed to assume infinite values. Finally, a glance at the graph of $Q(E)$ in Figure 1 shows that for large $h>0$ the binding energies tend to the set $\left\{\epsilon_{n}\right\}$, i.e., to the energies associated to the discontinuities of $Q(E)$.

\subsection{2. $h=+\infty$}

Let us now consider the last remaining possibility for a parity invariant (even) Coulomb interaction, namely, the impermeable case given by the choice $h^{+}=h^{-}=h=+\infty$ in (22). As we mentioned before, this is equivalent to imposing Dirichlet b.c. [7]

$$
\psi\left(0^{ \pm}\right)=0
$$

with $\tilde{\phi}\left(0^{ \pm}\right)$assuming arbitrary values. By using the expressions (A6) and (A8) from the Appendix 2, Equation (44) can be rewritten as

$$
B_{ \pm} W_{0}(E)=0,
$$

which results in non-trivial solutions for $B_{ \pm}$if, and only if, the binding energies satisfy $W_{0}(E)=0$, that is, the eigenvalues of energy are given by (39). Similarly to the previous case, conditions (45) with $W_{0}(E)=0$ allow arbitrary values for the coefficients $B_{ \pm}$, resulting in doubly degenerated energy eigenvalues and, as before, the pair of eigenfunctions associated with each $\epsilon_{n}$ can be chosen to be formed by an even and an odd eigenfunction.

Finally, we observe that the results for the case being considered here can be obtained as the limit $h \rightarrow+\infty$ of the previous case, subsection 4.2.1. However, we emphasize that the present case cannot be obtained from the results in the previous subsection by taking the limit $h \rightarrow-\infty$, since this would imply that the ground state energy tends to $E=-\infty$, violating the requirement $W_{0}(E)=0$ [notice that $\lim _{E \rightarrow-\infty} W_{0}(E)=1$ as seen in (A12)].

\section{CONCLUSION}

We investigated the one-dimensional hydrogen atom, with an $1 \mathrm{D}$ Coulomb interaction given by (3), by extending the distributional method, introduced in Calçada et al. [30] for contact interactions, to treat long-range interactions exhibiting a point singularity. After showing that the non-integrable singularity of the potential at the origin renders the ordinary Schrödinger equation illdefined, we introduced a distributional Schrödinger equation, Equation (5), with an interaction distribution $S[\psi, V]$ to be determined from the fundamental physical and mathematical requirements R1-R4, which follow from the general structure of quantum mechanics (section 2.2)-these requirements are expected to be satisfied by any interaction. Requirements R1 $\mathbf{R} 4$ allowed us to define the interaction rigorously-no ill-defined

TABLE 1 | Bound state energies and properties under parity of the corresponding eigenstates, for all subfamilies of parity invariant (even) attractive Coulomb interactions.

\begin{tabular}{llll}
\hline Interaction & Bound states energies & Eigenstates & Ground state \\
\hline $\begin{array}{l}\text { Permeable } \\
b \neq 0\end{array}$ & $\begin{array}{l}Q(E)=\frac{a+1}{b} \\
Q(E)=\frac{a-1}{b}\end{array}$ & $\begin{array}{l}\text { odd } \\
\text { even }\end{array}$ & $\begin{array}{l}\text { Finite energy } \\
\text { (even) (odd) if }(b>0)(b<0)\end{array}$ \\
\hline $\begin{array}{l}W_{0}(E)=0 \\
\text { Permeable }\end{array}$ & odd (even) if $a=+1(a=-1)$ & Finite energy \\
$a=0$ & $Q(E)=\frac{c}{2 a}$ & even (odd) if $a=+1(a=-1)$ & even (odd) if $a=+1(a=-1)$ \\
\hline Impermeable & $Q(E)=h$ & Doubly degenerated & Finite energy \\
$h$ real and finite & & Doubly degenerated \\
Impermeable & (even and odd eigenstates) & (even and odd eigenstates) \\
(Dirichlet) & $W_{0}(E)=0$ & Doubly degenerated & Finite energy \\
$h=+\infty$ & & (even and odd eigenstates) & Doubly degenerated \\
(even and odd eigenstates)
\end{tabular}

The binding energies associated to the zeros of $W_{0}(E)$ are given by Equation (39). For permeable origin interactions, the parity alternates between successive bound states. 
steps or infinities ever appear-and to restrict the interaction to a long range term outside the origin plus a family of contact interactions at the origin. The contact terms are obtained up to a four-parameter family of contact interactions, for the case of a penetrable origin, or up to a two-parameter family of interactions in the case of an impenetrable origin, in complete agreement with the SAE method (see, e.g., [7] and references therein).

The requirements $\mathbf{R} \mathbf{1}-\mathbf{R} \mathbf{4}$ are not enough to completely specify the interaction and, in particular, cannot determine the specific b.c. at the origin-additional physical input is necessary to that end. As it is well-known, symmetry is an excellent guide in these circumstances. Therefore, since the Coulomb potential is even under parity transformations, it is natural to require that the interaction distribution $S[\psi, V]$ associated with this potential also be invariant (even) under parity. We stress that the invariance of the Coulomb potential $\left(V(x)=\frac{\gamma}{|x|}, x \neq 0\right)$ under parity transformations is not sufficient to ensure that the interaction will be even under parity-information about the contact term is crucial to address this issue. In this context, it should be noticed that by providing an explicit form for $S[\psi, V]$, the distributional approach proves to be particularly adequate to deal with symmetry transformations. We have taken advantage of the explicit expression for the most general Coulomb distribution (i.e., the interaction distribution) to prove, in section 3, that the long-range term $S_{0}[\psi, V]$ is even under parity transformations. In this way, we concluded that the parity invariance of the total Coulomb interaction can be ensured if, and only if, the contact term also is invariant under parity transformations. As a consequence, we have shown that the additional requirement of parity invariance further reduces the possible contact terms associated with the generalized Coulomb interaction to a two-parameter family [26] (penetrable origin case) or a one-parameter interaction (impenetrable case). This, of course, still leaves considerable freedom in the choice of the b.c. at the origin, leading to several possible extensions of the Coulomb interaction-to further specify the interaction, additional input from the experimental situation must be considered [26, 34].

After giving a complete characterization of the subfamily of all parity invariant (attractive) Coulomb interactions, we conducted a systematic study of the bound states for all possible interactions in this subfamily. A summary of the results obtained is presented in Table 1, for convenience. From our analysis it follows that for parity invariant Coulomb interactions:

(i) the ground state energy is always finite;

(ii) for permeable interactions, the bound state energies are always non degenerated and the corresponding

\section{REFERENCES}

1. Loudon R. One-dimensional hydrogen atom. Am J Phys. (1959) 27:649-55. doi: 10.1119/1.1934950

2. Ruder H, Wunner G, Herold H, Geyer F. Atoms in Strong Magnetic Fields: Quantum Mechanical Treatment and Applications in Astrophysics and Quantum Chaos. Berlin: Springer Verlag (1994). eigenfunctions have definite parity (which alternates between even and odd for successive bound states).

(iii) for impermeable interactions, all the bound states energies are doubly degenerated and, for each binding energy, the corresponding pair of eigenfunctions can be chosen to have a definite parity (with one degenerate eigenfunction even and the other odd).

Thus, our results, obtained from a mathematically welldefined treatment of the 1D Hydrogen atom, clarify the highly controversial issues concerning the boundedness of the ground state and the parity as well as the degenerescence of the bound states (see [6] and references therein).

Finally, this work demonstrates that the method developed in Calçada et al. [30] for contact interactions can be generalized for long-range interactions having point singularities (in fact, it makes essential use of the results derived in Calçada et al. [30]). The distributional approach proves to be particularly suited for symmetry analyses (see also [31]), in addition to being a physically appealing alternative to SAE methods, and it could be used to investigate other one-dimensional singular interactions such as the $1 / x^{2}$ potential. In particular, for odd interactions (such as the $1 / x$ potential) the approach here developed specifies completely the interaction, since there exists no $1 \mathrm{D}$ odd contact interaction [30]. Finally, an important open problem is the generalization of the distributional approach to higher dimensions.

\section{DATA AVAILABILITY}

All datasets generated for this study are included in the manuscript and/or the supplementary files.

\section{AUTHOR CONTRIBUTIONS}

All the authors participated in the formulation of the problem and in the calculations. JL and LM wrote the manuscript. MC, $\mathrm{WM}$, and MP revised the text.

\section{FUNDING}

WM was fully supported by a fellowship from CAPES, a Brazilian agency.

\section{ACKNOWLEDGMENTS}

The authors would like to thank the referees for bringing references $[12,13,15,16]$ to our attention.
3. Bartnik AC, Efros AL, Koh WK, Murray CB, Wise FW. Electronic states and optical properties of PbSe nanorods and nanowires. Phys Rev B. (2010) 82:195313. doi: 10.1103/PhysRevB.82.195313

4. Downing CA, Portnoi ME. One-dimensional Coulomb problem in Dirac materials. Phys Rev A. (2014) 90:052116. doi: 10.1103/PhysRevA.90.052116

5. Ferré G, Astrakharchik GE, Boronat J. Phase diagram of a quantum Coulomb wire. Phys Rev B. (2015) 92:245305. doi: 10.1103/PhysRevB.92.245305 
6. Loudon R. One-dimensional hydrogen atom. Proc R Soc A Math Phys Eng Sci. (2016) 472:20150534. doi: 10.1098/rspa.2015.0534

7. de Oliveira CR, Verri AA. Self-adjoint extensions of Coulomb systems in 1, 2 and 3 dimensions. Ann Phys. (2009) 324:251-66. doi: 10.1016/j.aop.2008.06.001

8. Jaramillo B, y Romero RPM, nez Yépez HNN, Salas-Brito AL. On the one-dimensional Coulomb problem. Phys Lett A. (2009) 374:150-3. doi: 10.1016/j.physleta.2009.10.073

9. Haines LK, Roberts DH. One-dimensional hydrogen atom. Am J Phys. (1969) 37:1145-54. doi: 10.1119/1.1975232

10. Gomes JF, Zimerman AH. One-dimensional hydrogen atom. Am J Phys. (1980) 48:579-80. doi: 10.1119/1.12067

11. Itzykson C, Zuber JB. Quantum Field Theory. New York, NY: McGraw-Hill (1980).

12. Shang Y. Laplacian estrada and normalized laplacian estrada indices of evolving graphs. PLoS ONE. (2015) 10:e0123426. doi: 10.1371 /journal.pone. 0123426

13. Shang Y. Lower bounds for gaussian estrada index of graphs. Symmetry. (2018) 10:325. doi: 10.3390/sym10080325

14. de Oliveira CR, Verri AA. Mathematical predominance of Dirichlet condition for the one-dimensional Coulomb potential. J Math Phys. (2012) 53:052104. doi: $10.1063 / 1.4719976$

15. Golovaty Y. 1-D Schrödinger operators with coulomb-like potentials. arXiv: 190107218v2 (2019).

16. Savchuk AM, Shkalikov AA. Sturm-Liouville operators with singular potentials. Math Notes. (1999) 66:741-53.

17. Gordeyev AN, Chhajlany SC. One-dimensional hydrogen atom: a singular potential in quantum mechanics. J Phys A Math Gen. (1997) 30:6893.

18. Palma G, Raff U. The one-dimensional hydrogen atom revisited. Can J Phys. (2006) 84:787-800. doi: 10.1139/p06-072

19. Abramovici G, Avishai Y. The one-dimensional Coulomb problem. J Phys A Math Theor. (2009) 42:285302. doi: 10.1088/1751-8113/42/28/285302

20. Gebremedhin DH, Weatherford CA. Calculations for the one-dimensional soft Coulomb problem and the hard Coulomb limit. Phys Rev E. (2014) 89:053319. doi: 10.1103/PhysRevE.89.053319

21. Carrillo-Bernal MA, Núñez Yépez HN, Salas-Brito AL, Solis DA. Comment on "Calculations for the one-dimensional soft Coulomb problem and the hard Coulomb limit". Phys Rev E. (2015) 91:027301. doi: 10.1103/PhysRevE.91.027301

22. Gebremedhin DH, Weatherford CA. Reply to "Comment on 'Calculations for the one-dimensional soft Coulomb problem and the hard Coulomb limit". Phys Rev E. (2015) 91:027302. doi: 10.1103/PhysRevE.91.027302

23. Ivetić B. On the symmetry of a one-dimensional hydrogen atom. J Math Phys. (2018) 59:102106. doi: 10.1063/1.5028500

24. Kurasov P. On the Coulomb potential in one dimension. J Phys A Math Gen. (1996) 29:1767.

25. Kurasov P. Response to "Comment on 'On the Coulomb potential in one dimension"' by Fischer, Leschke and Müller. J Phys A Math Gen. (1997) 30:5583.
26. Tsutsui I, Fülöp T, Cheon T. Connection conditions and the spectral family under singular potentials. J Phys A Math Gen. (2003) 36:275. doi: 10.1088/0305-4470/36/1/319

27. Coutinho FAB, Amaku M. Renormalizing the kinetic energy operator in elementary quantum mechanics. Eur J Phys. (2009) 30:1015. doi: 10.1088/0143-0807/30/5/010

28. Fülöp T. Singular potentials in quantum mechanics and ambiguity in the self-adjoint hamiltonian. SIGMA. (2007) 3:107. doi: 10.3842/SIGMA.2007.107

29. Fischer W, Leschke $H$, Müller $P$. The functional-analytic versus the functional-integral approach to quantum Hamiltonians: the one-dimensional hydrogen atom. J Math Phys. (1995) 36:2313-23. doi: 10.1063/1.531040

30. Calçada M, Lunardi JT, Manzoni LA, Monteiro W. Distributional approach to point interactions in one-dimensional quantum mechanics. Front Phys. (2014) 2:23. doi: 10.3389/fphy.2014.00023

31. Lee MA, Lunardi JT, Manzoni LA, Nyquist EA. Double general point interactions: symmetry and tunneling times. Front Phys. (2016) 4:10. doi: 10.3389/fphy.2016.00010

32. Zemanian AH. Distribution Theory and Transform Analysis: An Introduction to Generalized Functions, with Applications. New York, NY: Dover (1987).

33. Kurasov P. Distribution theory for discontinuous test functions and differential operators with generalized coefficients. J Math Anal Appl. (1996) 201:297-323.

34. Fischer W, Leschke H, Müller P. Comment on 'On the Coulomb potential in one dimension’ by P. Kurasov. J Phys A Math Gen. (1997) 30:5579.

35. Vindas J, Estrada R. Distributionally regulated functions. Stud Math. (2007) 181:211-36. doi: 10.110.4064/sm181-3-2

36. Łojasiewicz S. Sur la valeur et la limite d'une distribution en un point. Stud Math. (1957) 16:1-36.

37. Campos Ferreira J. Introduction to the Theory of Distributions. Essex: Addison Wesley Longman (1997).

38. Albeverio S, Dąbrowski L, Kurasov P. Symmetries of schrödinger operators with point interactions. Lett Math Phys. (1998) 45:33.

39. Schiff LI. Quantum Mechanics. 3rd ed. New York, NY: McGraw-Hill (1968).

40. Abramowitz M, Stegun IA. Handbook of Mathematical Functions with Formulas, Graphs, and Mathematical Tables. New York, NY: Dover (1965).

Conflict of Interest Statement: The authors declare that the research was conducted in the absence of any commercial or financial relationships that could be construed as a potential conflict of interest.

Copyright (C) 2019 Calçada, Lunardi, Manzoni, Monteiro and Pereira. This is an open-access article distributed under the terms of the Creative Commons Attribution License (CC BY). The use, distribution or reproduction in other forums is permitted, provided the original author(s) and the copyright owner(s) are credited and that the original publication in this journal is cited, in accordance with accepted academic practice. No use, distribution or reproduction is permitted which does not comply with these terms. 


\section{APPENDIX: BASIC NOTIONS ON SCHWARTZ'S DISTRIBUTION THEORY}

In this Appendix, for convenience of the reader, we briefly present some of the concepts of the Schwartz distribution theory that are directly necessary for an understanding of the main text. For an extensive presentation of distribution theory we refer to Zemanian [32].

A distribution $f$ is a continuous linear (complex) functional on a space of test functions. Here we will consider the test functions space as the space $\mathcal{D}_{0}$ formed by all the (complexvalued) functions $\varphi(x)$, defined on the entire real line, which are infinitely smooth and have compact support (i.e., they vanish outside some finite interval, which does not need to be the same for all the test functions).

Let $\varphi$ be a test function, and $f$ be a distribution. The complex number associate to $\varphi$ by the distribution $f$ is denoted by $\langle f, \varphi\rangle$. If $f(x)$ is a locally integrable function (i.e., a function which is Lebesgue integrable over every finite interval), then the distribution $f$ associated to the function $f(x)$ is said to be regular, and it is given by

$$
\langle f, \varphi\rangle=\int_{-\infty}^{\infty} f(x) \varphi(x) d x
$$

for any test function $\varphi$. Distributions that can not be associated in this way to locally integrable functions are said to be singular - a well-known example is the Dirac delta functional $\delta$, defined by $\langle\delta, \varphi\rangle=\varphi(0), \forall \varphi$. When a distribution is regular we will not distinguish between the function and the regular distribution associated to it, and we will often use the abusive notation $f(x)$ to refer to the distribution $f$ (even for singular distributions).

Any distribution can be infinitely differentiated. Denoting the $n$-th derivative of a test function or a distribution by a superscript (n), we have

$$
\left\langle f^{(n)}, \varphi\right\rangle \equiv(-1)^{n}\left\langle f, \varphi^{(n)}\right\rangle
$$

for all test functions $\varphi$. Every distribution has primitives (indefinite integrals), and any two primitives of a given distribution differ by a constant. We denote the primitive of a distribution or test function by the superscript $(-1)$. Considering a fixed test function $\varphi_{0}$ satisfying $\int_{-\infty}^{\infty} \varphi_{0}(x) d x=1$, a primitive of $f$ is a distribution $f^{(-1)}$ defined as

$$
\left\langle f^{(-1)}, \varphi\right\rangle \equiv\langle c, \varphi\rangle-\langle f, \psi\rangle, \quad \forall \varphi
$$

where $c$ is an arbitrary constant, $\psi(x)=\int_{-\infty}^{x} \chi(t) d t$ and $\chi(x)=\varphi(x)-\varphi_{0}(x) \int_{-\infty}^{\infty} \varphi(t) d t$.

On any closed finite interval $I$ every distribution $f$ can be written as the $(r+2)$-th order derivative of a distribution $h$ associated to a continuous function whose derivative is not continuous in this interval (in the ordinary sense) - see [32], p. 162. In other words, $\langle f, \varphi\rangle=\left\langle h^{(r+2)}, \varphi\right\rangle$, for all $\varphi$ with support in $I$. The integer $r$ is the order of the distribution $f$. Therefore, if $r=-2$ the distribution $f$ corresponds to a continuous function whose derivative is not continuous on the interval $I$; if $r=-1, f$ is the derivative of a continuous distribution, but $f$ is not continuous; if $r=0, f$ is not locally integrable and corresponds to a singular distributon (since $f^{(-1)}$ is not continuous). Summarizing, $r \leq-2$ characterizes distributions that correspond to continuous functions (regular distributions), $r \geq 0$ characterizes singular distributions, and distributions with $r=-1$ may be either singular or regular. If $r=-\infty$ the distribution is infinitely smooth on $I$ (see [32], p. 162, for details). When the order is finite, differentiation increases the order by one, whereas indefinite integration decreases the order by one. The order of a finite sum of distributions is the maximum order among the various terms, except when the distributions of largest order cancel each other, resulting a lower order for the sum [32].

Two distributions $f$ and $g$ are said to be equal on an open set $I$ if they associate the same number $\langle f, \varphi\rangle=\langle g, \varphi\rangle$ to every test function $\varphi$ whose support is contained in $I$ (if $I=\mathbb{R}$ then the two distributions are simply said to be equal). Two functions that are locally integrable and differ on a set of zero Lebesgue measure define the same distribution. Thus, the same regular distribution is associated to the class of all locally integrable functions which differ among themselves only on a set of zero Lebesgue measure.

Any distribution $f$ can be multiplied by an infinitely smooth function $\eta(x)$, resulting in a new distribution $\eta f$, according to $\langle\eta f, \varphi\rangle \equiv\langle f, \eta \varphi\rangle$. If $f$ and $g$ are two locally integrable functions such that their product $f g$ is also locally integrable, then the product of the corresponding regular distributions exists, and it is the regular distribution defined as

$$
\langle f g, \varphi\rangle \equiv \int_{-\infty}^{\infty} f(x) g(x) \varphi(x) d x, \quad \forall \varphi .
$$

However, it is an important fact of Schwartz's distribution theory that the product of two arbitrary distributions cannot be defined in a unique way. For example, the function $f(x)=\frac{1}{\sqrt{|x|}}$ is locally integrable and thus defines a regular distribution. The function $g(x)=\frac{1}{|x|}$, on the other hand, is not integrable on any interval including the origin. Therefore, the product $f^{2}(x)=$ $g(x)$ does not define a regular distribution (in addition, one can define several non-equivalent singular distributions which coincide with $\frac{1}{|x|}$ over any interval which does not include the origin).

The following Theorem and its corollary are of crucial importance in this work.

Theorem. Zemanian ([32], p. 98) A necessary and sufficient condition for a distribution $f(x)$ on $\mathbb{R}$ to have a support consisting of a single point $x_{0}$ is that it be a finite sum

$$
f(x)=\sum_{n=0}^{r_{m}} a_{n} \delta^{(n)}\left(x-x_{0}\right),
$$

where the $a_{n}$ are constants, $a_{r_{m}} \neq 0$, and $r_{m}$ is the singular order of the distribution $f$. 
As a corollary, it follows that if two distributions $f$ and $g$ are equal on the open region $\mathbb{R} \backslash\{0\}$, then their difference $f-g$ must have its support concentrated at a single point (the origin), and it is a finite linear combination of the delta distribution and its derivatives.

\section{APPENDIX: BOUND STATES OF THE 1-D HYDROGEN ATOM}

Here we present some properties of the (well-known) bound state solutions of the Schrödinger equation for the 1D Hydrogen atom, given by (6), on the disjoint union $(-\infty, 0) \cup(0,+\infty)$. By performing the change of variable $z=\sqrt{-4 E}|x|, x \neq 0$, Equation (6) becomes a special case of the Whittaker equation, namely [40]

$$
\beta^{\prime \prime}(z)+\left(\frac{\tilde{\gamma}}{z}-\frac{1}{4}\right) \beta(z)=0, \quad z \neq 0,
$$

with $\tilde{\gamma}=\frac{-\gamma}{\sqrt{-4 E}}$. For bound states $(E<0)$, both $z$ and $\tilde{\gamma}$ are real and positive (since in this work we are considering only the attractive Coulomb potential, $\gamma<0$ ). The two linearly independent solutions of Whittaker's equation, Equation (A2), are [40]

$$
\begin{aligned}
& \mathcal{M}_{\tilde{\gamma}, \frac{1}{2}}(z)=z \mathrm{e}^{-\frac{z}{2}} M(1-\tilde{\gamma}, 2, z), \\
& \mathcal{W}_{\tilde{\gamma}, \frac{1}{2}}(z)=z \mathrm{e}^{-\frac{z}{2}} U(1-\tilde{\gamma}, 2, z)
\end{aligned}
$$

where $M(a, b, z)$ and $U(a, b, z)$ are the confluent hypergeometric functions of the first and of the second kind, respectively.

For negative energies $\mathcal{M}_{\frac{-\gamma}{\sqrt{-4 E}}, \frac{1}{2}}(\sqrt{-4 E}|x|)$ is not an acceptable solution, since it diverges when $|x| \rightarrow \infty$. Therefore, the general solution of (A2) for bound states is

$$
\psi(x)=\mathcal{W}_{\frac{-\gamma}{\sqrt{-4 E}}, \frac{1}{2}}(\sqrt{-4 E}|x|)\left[B_{-} \theta(-x)+B_{+} \theta(x)\right],
$$

where $\theta(x)$ is the Heaviside theta function and the arbitrary constants $B_{ \pm}$must be related by the boundary conditions at the origin, which depend on the specific choice of the parameters determining the interaction (see section 2.2). It follows from the properties of the Whittaker functions (see [40], Ch. 13) that $\psi(x)$ has finite limits at both sides of the origin, namely

$$
\psi\left(0^{ \pm}\right)=B_{ \pm} \mathcal{W}_{\frac{-\gamma}{\sqrt{-4 E}}, \frac{1}{2}}(0)=B_{ \pm} W_{0}(E)
$$

where we have defined

$$
W_{0}(E) \equiv \frac{1}{\Gamma\left(1+\frac{\gamma}{\sqrt{-4 E}}\right)} .
$$

In section 2 the function $\tilde{\phi}(x)$, Equation (12), was introduced and shown to have finite lateral limits at both sides of the origin. From (A5), these lateral limits can be explicitly obtained as

$$
\tilde{\phi}\left(0^{ \pm}\right)= \pm B_{ \pm} F_{0}(E)
$$

with

$$
\begin{aligned}
& F_{0}(E)=W_{0}(E) Q(E), \\
& Q(E)=\left[\frac{1}{2} \gamma \log (-4 E)+\gamma \psi^{(0)}\left(\frac{\gamma}{2 \sqrt{-E}}\right)+2 \gamma \gamma+\sqrt{-E}\right],
\end{aligned}
$$

where $\gamma$ is the Euler's constant and $\psi^{(0)}(x)$ is the digamma function, defined as $\psi^{(0)}(x)=\frac{1}{\Gamma(x)} \frac{d}{d x} \Gamma(x)$ [40].

The functions $W_{0}(E), F_{0}(E)$ and $Q(E)$ have the following important properties, which are used in the main text of the paper.

- $W_{0}(E)$ is continuous and has simple zeros at the points in which the gamma function in (A7) diverges, namely at

$$
E=\epsilon_{n}=-\frac{\gamma^{2}}{4 n^{2}}, \quad n=1,2,3, \cdots
$$

- $F_{0}(E)$ is continuous, and at the zeros of $W_{0}(E)$ it assumes the nonzero values

$$
F_{0}\left(\epsilon_{n}\right)=(-1)^{n}(n-1) ! \gamma, \quad n=1,2,3, \cdots .
$$

- $Q(E)=\frac{F_{0}(E)}{W_{0}(E)}$ is continuous in any interval $E \in\left(\epsilon_{n}, \epsilon_{n+1}\right)$, with $n$ a positive integer. At the boundaries of these intervals $Q(E)$ diverges.

- In the limit $E \rightarrow-\infty$ we have

$$
\begin{aligned}
& \lim _{E \rightarrow-\infty} W_{0}(E)=1, \\
& \lim _{E \rightarrow-\infty} Q(E)=-\infty, \\
& \lim _{E \rightarrow-\infty} F_{0}(E)=-\infty .
\end{aligned}
$$

